\title{
Health Information Integration Model Design Based on Mobile Medical
}

\author{
Shu Lii ${ }^{\mathrm{a}}$, Na Liu ${ }^{\mathrm{b}, *}$ \\ Center of Health Administration and Development studies, Hubei University of Medicine, Shiyan 442000, \\ China. \\ a345619552@qq.com, b34703558@qq.com \\ *corresponding author
}

Keywords: Information Integration, Process Reengineering, Mobile Medical.

\begin{abstract}
With the rapid development of wearable devices, mobile APP applications and emerging network technologies, mobile medical service has been realized, which has had a great impact on traditional medical care, and improved the efficiency and quality of medical services. In this paper, the systematic reform method is used to reform and optimize the two traditional processes of registration, interrogation and health management. Based on mobile medical, we design the health information integration model contains the wisdom of hospital sub module, family health die block and regional health sub module.
\end{abstract}

\section{Introduction}

With the emergence and development of the concept of smart medical, mobile medical as an important part of intelligent medical care, set off a wave of research and development boom. Mobile medical service is to provide medical service by using mobile communication technology, including medical information service, telemedicine service, expert reservation service, hospital information mobile solution and so on ${ }^{[1]}$. Many mobile medical devices and software for monitoring came into being, human health bracelet, for registration, see a mobile phone software, change the traditional medical process. Based on the introduction of mobile medical technology, the traditional medical process is discussed, analysed and optimized.

\section{Mobile Medical Technologies}

The strong development of mobile healthcare has benefited from the rapid progress and extensive application of related technologies, including wearable devices, mobile APP applications, emerging networking technologies and other aspects of ${ }^{[1]}$.

\subsection{Wearable Devices}

Wearable devices that can be used in mobile medical services can be divided into two categories: for patients and for ordinary users. The former is used for monitoring the patient's physiological state, including real-time monitoring of the patient's heart rate, breathing, blood pressure, blood glucose, body temperature is closely related to the physical condition of patient's data; the latter detection of moving ordinary user status, calorie consumption and other data, is convenient for the user to manage the health of their time. Wearable devices support access to wireless networks, and then upload data to the analysis centre or mobile phones such as mobile clients.

\subsection{Related APPs}

The existing mobile APP applications for medical services can meet the different needs of different users. For example, online inquiry APP spring doctor, mobile platform provides online information inquiry, patients and medical staff to make timely and effective communication in APP; 
make an appointment APP micro medicine, hospital or WeChat public number, the patients at home to make an appointment, free queue time long traditional process APP; the medicine information inquiry "medication assistant, providing drug information query convenient for clinicians, pharmacists and medical personnel ${ }^{[2]}$.

\subsection{Emerging Network Technologies}

The massive expansion of mobile terminals, such as mobile phones and tablet PCs, has greatly promoted the development of wireless networking technology. Wireless technology provides strong technical support for mobile health care. Today, users can use WiFi wireless access or communication services provided by the $4 \mathrm{G}$ network, access to the network at any time, and the cost is cheap.

\section{Impact of Mobile Healthcare on Medical Process Reengineering}

Business process reengineering (BPR) is an improved philosophy and thought. Its goal is to maximize the added value of these processes by minimizing the added value of the business processes of the organization, and thus to achieve a leap in performance improvement ${ }^{[3]}$. The application of mobile medical service has a great impact on traditional medical treatment, which subverts some of the traditional medical business processes and greatly improves the efficiency and quality of medical services. In this process, it is necessary to change the traditional medical process. There are two basic modes of the transformation process: one is the transformation of law system, the analysis and understanding of the existing process system by creating a new process based on the existing process; two is a new design method, rethink the way in providing products or services from the root, on a piece of paper to the design process of ${ }^{[3]}$. In this paper, the systematic reform method is used to reform and optimize the registration inquiry process and the health management process.

\subsection{Process Reengineering of Registration Inquiry}

Traditional registration inquiry process requires patients to go to the hospital window, select the appropriate department registration, after registration to the corresponding department, triage desk for triage, triage to the doctor's individual, and then wait in line for interrogation. In this process two times for the appearance and reached the number of hours, a lot of unnecessary consumption of energy and resources.

The advent of mobile medicine can revolutionize this situation. The use of mobile medical APP software, users can even provide one or two days ahead of the remote login medical services platform, query doctors information, select the appropriate departments and doctors, in the online registration and use online banking to pay the registration fee; in the hospital system, can be realtime detection to the network registered patients, the patients in time online diagnosis and arranging, and real time to push patients, there are several patients waiting time, so that the patients choose the appropriate location for treatment time. The improved process saves the patients a great deal of waiting time, realizes the triage and improves the crowding situation in hospitals and other medical service places.

\subsection{Process Design of Health Management}

For patients with chronic diseases and ordinary users of home support therapy, good health management can be achieved with mobile health care systems and good docking with health care providers.

The physiological status of the user wearable device for real-time monitoring of users, especially for patients with chronic disease or other diseases need to closely monitor patients, wearable devices to access the wireless network, at any time the user physiological data uploaded to the user of the mobile terminal to monitor the installation in the mobile medical APP, as users of health management data analysis when the normal range of sources; a health data index exceeds the set when the wearable device beeps or vibration effect, to remind the user, the user can adjust itself and 
turn off the sound; when the normal range of a health data set more than the serious, and not on the users of wearable devices to make the tone during the reaction, the mobile terminal APP to implement alarm functions, to set the number of emergency telephone calls good, including the user's relatives and 120 emergency The telephone, to play pre-recorded voice at the same time, the use of mobile positioning function of the terminal to send user location of medical services; medical institutions received a phone call and location, can promptly dispatched ambulances and medical rescue team.

Mobile health played an important role in the health management process, the status quo has changed the traditional medical procedures in patients unable to timely help, the hospital can't be timely sent a rescue team; at the same time, ordinary users of health data collection and storage, provide the basis for strong support for the analysis of large data later.

\section{Design of the Health Information Integration Model}

Emerging health service model by adopting new type of sensor, the Internet of things, such as communication technology combined with modern medicine idea, build on the basis of personal health electronic medical records, combined with the regional health resources information management platform, in order to achieve optimization of regional health resources allocation, shorten the sufferings patient flow, reduce relevant formalities, convenient information transfer target. Cooperate with each other three parts of the model of collaboration, to achieve the ultimate goal of medical wisdom. Through the several means of circulation data shown in figure 1, including signs data information, treatment, health care, health information management, can understand the operation mechanism of health service model. 


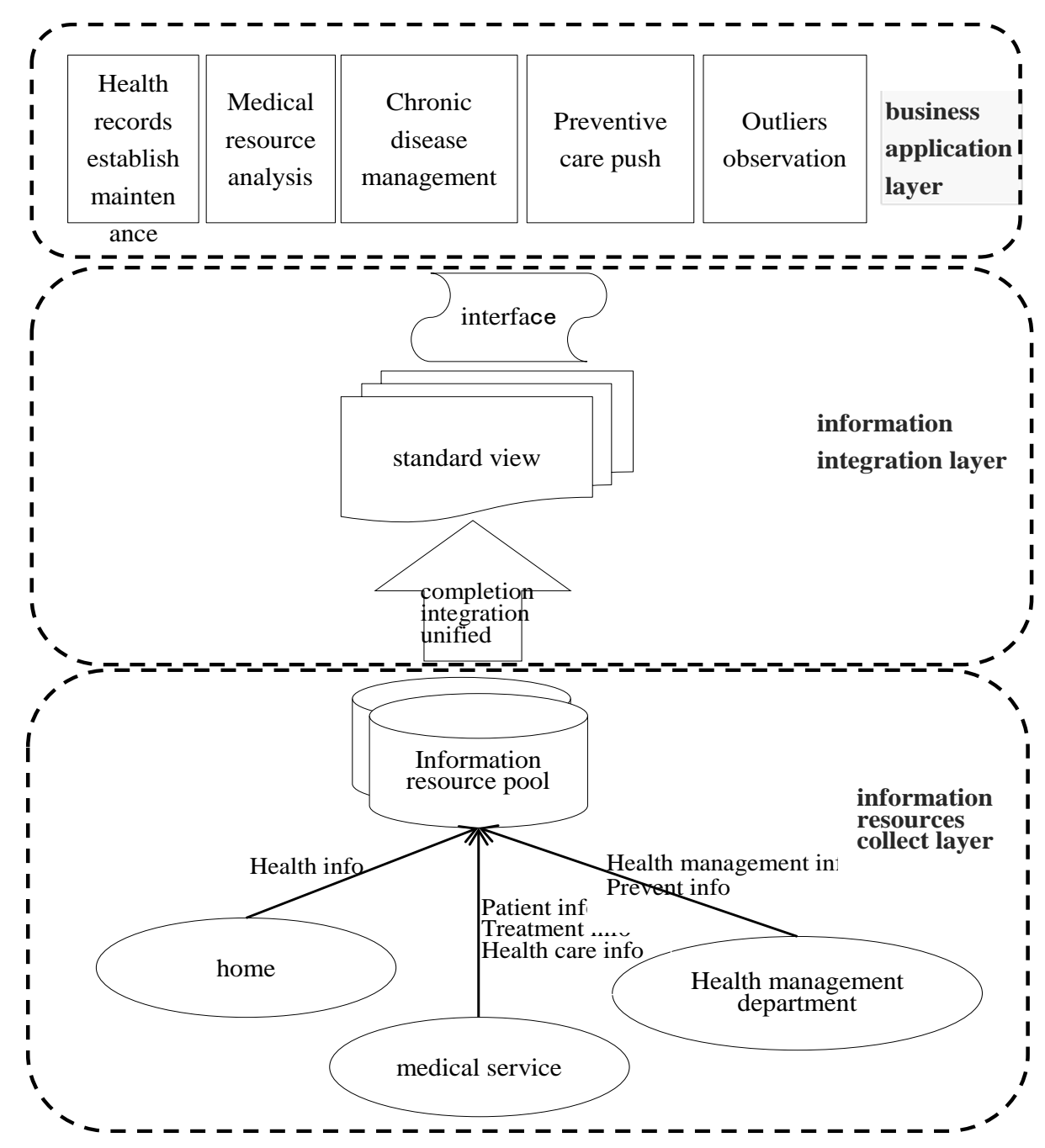

Fig.1 Health Information Integration Model

\subsection{Signs Data}

Signs statistics data are collected by family healthy child module, through the portable equipment monitoring data to the signs, such as temperature, pulse, exercise, etc., access the Internet or mobile APP uploaded to the system.

The signs data has 3 affects. First of all, the user is used for self-control and health management, ordinary users can undertake health movement observation data, chronic diseases patients by monitoring physiological data real-time monitoring condition; Second, when signs of abnormal data, automatic transmission to wisdom hospital son module, the hospital received data can be treated in a timely manner to contact, to realize the efficient of remote medical treatment; Third, family health data uploaded to regularly regional health son module, the database for a regional health management department set up health, can effective judgment or projections for regional high incidence of disease, the residents' health records maintenance area.

\subsection{Treatment Information}

Treatment subsystem, information generated by wisdom hospital during the period of the treatment of patients, the disease type, treatment time, treatment, treatment effect and so on data synchronization subsystem to family health. Treatment by intelligent hospital information subsystem is passed to the family, in order to send out a follow-up treatment reminder, such as review the booking; Family health can timely calibration data, update family member's health 
records; By mastering information treatment, patients can be targeted to push related information such as health, disease prevention and health care.

\subsection{Health Data}

Health care data generated by the patients in the clinic process, may be associated with the process of hospital expense list, also may come from also in other health care services (such as pharmacy, etc.) to produce cost.

Health care data flow in the three child module, on the one hand, convenient for patients in a timely manner the expense deduction and reimbursement, through process improvement, for the high cost of medical treatment of a serious illness, as long as the condition, to submit an expense account, over the treatment slows down the patient's payment pressure; On the other hand, the administrative departments for public health control of health care data and information, through the analysis of data, is advantageous to the management of regional health resources distribution, particularly in the data can meet the diagnosis and treatment of execution, facilitate timely find problems, solve the problem.

\subsection{Health Management Information}

Wisdom hospital son module, there is a lot of macro level to treat disease information, and the treatment of vital information focus, focus on an area health management information of medical institutions in a period of statistics, including the cycle of a high incidence of disease, neonatal vaccine in macro information, etc. Health authorities through the macro statistics, can timely send the medical service institutions a disease warning, send a targeted the family child modules electronic health, etc.

\section{Information Integration}

Health service model after redesign, the past information structure will be upset, want to make the new model is working well, need to integrate information and integration, unified management and use. Based on the standardization of information management, health service model of each module can be interactive, sharing information resources.

There are two roles of information integration: one is the accumulation of information, will be more child module of the collected data through integration of standardized database, including patient information, medical service information, treatment, health care, health information management and so on, all the entries are stored in which; Second, information access services, when information is accumulated to a certain degree, regional health management department can access the relevant data, analysis and mining, applied in many aspects, such as health management and decision-making, in order to realize the unification of the regional health resources distribution management.

Information integration framework consists of information resource collection layer, information integration, business application layer three parts. Information resource collection layer from the health service mode of the three sub modules, respectively, cell phone information, information accumulation, it is important to note the information gathering process only allows the accumulation of information, are not allowed to modify, and delete, so as not to destroy the original message. Information integration layer by establishing a standardized interface to the database in different fields and format integration, unity, completion, the formation of standardized view, and does not destroy the original table structure. Business application layer data mining personnel to access data in a standardized view, through different aspects carries on the analysis and mining, such as peak/outliers, observation and treatment when reach the line of warning information in time; Maintenance area residents' health records; Analysis of this regional institutions, medical resource utilization and so on, to better serve the regional medical and health management. 


\section{Summary}

This paper discusses the reengineering and optimization of traditional medical processes under the support of mobile medical technology. In the process of process optimization and design, the need to build APP mobile medical and health services platform, however, whether APP or mobile hospital information platform, there are many compatibility problems, for example, remote medical services are subject to the hospital and geographical constraints. In the future research, we can discuss how to integrate various support platforms into a unified platform to realize the sharing of resources.

\section{Acknowledgments}

This work was financially supported by Key Research Canter for Humanities and Social Sciences in Hubei Province (Hubei University of Medicine) (Project number: 2015ZD002).

\section{Reference}

[1]. Chen Xiaoping, Zhang Tao, Zong Hong. Design of Health Management System based on Mobile Medical Technology [J]. China Health Information Management Journal, 2015 (1) 25-28.

[2]. Yin Yawei. Preliminary Study of Mobile Medical Products from the Perspective of Product Service System [J]. Jintian, 2015 (2) 366-367.

[3]. Wang Pu, Cao Diefeng. Process Reengineering. Beijing, CITIC publishing house, 2005. 